\title{
Relación céntrica: revisión de conceptos y técnicas para su registro. Parte I
}

\section{Centric relation: concepts revision and recording techniques. Part I}

\section{Orozco Varo A*, Arroyo Cruz G*, Martínez de Fuentes R**, Ventura de la Torre J**, Cañadas Rodríguez D***, Jiménez Castellanos E****}

\begin{abstract}
RESUMEN
La relación céntrica ha sido objeto de disparidad de criterios en la odontología durante más de un siglo. A lo largo de este trabajo, vamos a exponer diferentes métodos usados tanto para obtener el registro, como para comprobar su certeza y corroborar la posición condilar.

Dado que no existe evidencia científica en el tema, en este trabajo, revisaremos los estudios experimentales que aparecen en la literatura. Los hemos divididos en dos partes, la primera relacionada con la definición de relación céntrica y la segunda parte relacionada con técnicas de registro, posición condilar y la discrepancia entre relación céntrica y oclusión céntrica.
\end{abstract}

Palabras clave: Relación céntrica, oclusión céntrica, técnicas de registro.

\section{SUMMARY}

The centric relation has been controversial on dentistry during more of one century. Along of this work, we are going to expose different methods to recording as checking the accuracy and condilar position. Given that there aren't scientific evidence, in this work, we review the researches publicated. We have divided them into two parts; the first is related with the definition of centric relation and the second part is related with recording techniques, condilar position and centric relation- centric occlusion discrepancy.

Key words: Centric relation, centric occlusion, recording techniques.

Fecha de recepción: 17 de marzo de 2008.

Aceptado para publicación: 15 de abril de 2008.

* Licenciada en Odontología. Colaboradora clínica de Prótesis Estomatológica. Facultad de Odontología. Universidad de Sevilla.

** Profesor asociado de Prótesis Estomatológica. Facultad de Odontología. Universidad de Sevilla.

*** Profesos titular de Prótesis Estomatológica. Facultad de Odontología. Universidad de Sevilla.

**** Catedrático de Prótesis Estomatológica. Facultad de Odontología. Universidad de Sevilla.

Orozco Varo A, Arroyo Cruz G, Martínez de Fuentes R, Ventura de la Torre J, Cañadas Rodríguez D, Jiménez Castellanos E. Relación céntrica: revisión de conceptos y técnicas para su registro. Parte I. Av. Odontoestomatol 2008; 24 (6): 365-368.

\section{INTRODUCCIÓN}

Uno de los términos sujetos a más controversia en la odontología en general y en la prostodoncia en particular, es la relación céntrica y no sólo en lo referente a su definición sino también al procedimiento para registrarla, labor en la que trabajaron numerosos autores con el objetivo de encontrar la manera más fiable de reproducir dicha posición.

En esta primera parte del trabajo revisamos la literatura relacionada con el concepto de relación céntrica. 


\section{CONCEPTO DE RELACIÓN CÉNTRICA}

Durante años, se ha discutido sobre la definición de relación céntrica $(\mathrm{RC})$.

Una de las definiciones más antiguas fue descrita por BB McCollum en 1939 (1), según la cual los cóndilos estaban localizados en una posición posterior en la fosa glenoidea. El propio McCollum en 1960 en su artículo "El eje bisagra mandibular y un método para localizarlo" mantiene la definición de posición más retruida del cóndilo en la fosa (2).

Lucía en "Relación Céntrica: teoría y práctica" de 1960, consideraba que cuando los centros de rotación condilar están en eje terminal bisagra hay RC (3).

En la primera publicación del Glosario de Términos Protodónticos (GTP) en 1956, la definición de RC concordaba con el concepto clásico predominante (4), pero a finales de los 70' esa relación condilar posterior fue cuestionada, la porción posterior de la ATM está totalmente vascularizada y contiene la fijación posterior del disco de manera que la propia morfología articular favorecería a un desplazamiento inferior del cóndilo si la mandíbula es empujada hacia atrás, así en 1987, en la $5^{\text {a }}$ edición del GTP, se definía la posición del cóndilo como anterior y superior en la fosa glenoidea (5). Esta última definición es la primera en el listado de definiciones de las ediciones del 1994,1999 y 2005 aunque seguida de varias definiciones también aceptadas (6-8).

Según Dawson en 1985, la RC es la relación de la mandíbula con el maxilar cuando los cóndilos están en la posición más superior contra la eminencia independientemente de la posición dentaria y dimensión vertical, posteriormente la amplió añadiendo el concepto de medial (9).

La últimas ediciones del GTP recogen también las definiciones de varios autores como son la de Ash (cóndilos y discos en la posición más medial y superior) y Ramsfjord (cóndilos en posición anterosuperior), ambas pronunciadas en comunicaciones personales en 1993.

Aún así, en la $4^{\text {a }}$ edición en español en 1996 de "Oclusión" de ambos autores, al hablar de RC vuel- ven a mencionar el concepto clásico de "posterior" (10).

En los GTP se recogen 7 definiciones diferentes de $\mathrm{RC}$, algunas incluso parecen contradecirse.

GJ.Christensen, en 2004, publicaba una definición muy clara: localización posterior más confortable de la mandíbula cuando es manipulada suavemente hacia atrás y arriba en una posición retrusiva. (11).

Otros autores, como S Davies y Gray en "¿Qué es oclusión?" de 2001, matizan la necesidad de definir la RC desde el punto de vista anatómico, conceptual y geométrico (12).

En lo que parece haber una mayor unificación de criterios es en la ausencia necesaria de contracturas musculares al tomar los registros, no debe haber participación muscular del paciente (Lucía, 1953; Calagna, 1973; Posselt, 1973; Cantor, 1972; Long, 1970; Ash y Ramfjord, 1984; Woelfel, 1986) (13-19).

Según Neff 1981 "la relación céntrica es usada como la posición inicial porque es la más estable y una de las más fáciles de reproducir, los cóndilos y las fosas se van continuamente remodelando a través del cambio de dentición temporal a permanente, esto es un intento de adaptación a las necesidades específicas de estas denticiones. Cuando es necesario tratar a un paciente con prótesis removible, los colocamos en relación céntrica ya que esta es la posición que puede reproducirse de forma más efectiva” (20).

Para autores como Neff la RC es una posición funcional, no forzada que se presenta en la deglución y masticación. Es una posición terminal que permite hacer registros y trasladarla a un articulador, es la única reproducible de forma sistemática. Sin embargo, otros muchos piensan que la RC es una posición bordeante o límite, una retrusión que no es natural ni fisiológica sino una posición extrema bordeante, basándose en esta creencia, Jankelson y cols. definen el término miocéntrica o posición céntrica generada por la musculatura, es decir, el cierre isotónico de la mandíbula desde una posición fisiológica de reposo hasta un contacto dentario con la musculatura en mínima actividad eléctrica. Ellos consideraban que normalmente se situaba entre RC y MI. Para 
llegar a dicha posición se ayudan de la neuroestimulación eléctrica transcutánea (TENS) que provoca una contracción rítmica, periódica e isotónica al ser aplicada sobre los V y VII pares craneales, más adelante comentaremos algunos estudios donde se usaron esta técnica y los resultados obtenidos $(21,22)$.

La permanencia de la relación intermaxilar en $\mathrm{RC}$ ha sido criticada durante años, tal y como recogen en "Neurofisiología de la Oclusión" los doctores Sencherman y Echeverri, esta idea se basaba en tres críticas fundamentalmente:

1. No es una relación no forzada puesto que hay que manipular la mandíbula del paciente. Para rebatirlo se proponen las técnicas de Long (23) o Lucía (24) entre otros con las cuales se evita ejercer fuerzas sobre el paciente.

2. En muchos casos, cuando el paciente se rehabilita haciendo coincidir RC con MI, al tiempo vuelven a aparecer dos relaciones diferentes. [Celenza, 1973 (25); Glickman y cols., 1974 (26)]. Actualmente se considera que cuando eso ocurre es porque no se había localizado la posición inicialmente.

3. La gran mayoría de pacientes presentan una MI que no coincide con la $\mathrm{RC}$, se asume pues que esa posición adquirida o habitual es la normal en el paciente. [Rieder, 1978 (27)]. Algunos autores consideran esa valoración ligera ya que algunas situaciones consideradas normales pueden ser patológicas o potencialmente patológicas (20).

Por otro lado, autores como Schuyler, 1935 o Mann y Pankey, 1963, apoyan una "céntrica larga" donde las prematuridades e interferencias oclusales sean eliminadas desde RC a MI $(28,29)$.

El glosario debería servir como diccionario de términos de oclusión pero las definiciones en la literatura son más extensas y además muchos autores usan una terminología propia lo cual aumenta la confusión de estudiantes y profesionales.

Esta inquietud llevó a Jasinevicius y sus colaboradores en 2000, a investigar sobre el consenso en la definición de RC encuestando a profesores y estudiantes de 7 escuelas dentales estadounidenses; la encuesta fue realizada por 117 profesores y 112 alumnos, se recopilaron datos de edad y año de gradua- ción, se les presentaban seis definiciones de RC recogidas en el GTP y se les preguntaba cual de ella usaban o le habían enseñado, también se les dejaba espacio para que ellos aportasen una. Los resultados mostraron que la controversia sigue vigente en este siglo. Las dos definiciones más seleccionadas fueron la más antigua (1956, posición posterior) y la de 1987, posición anterosuperior (1).

A pesar de toda la controversia, todavía la $\mathrm{RC}$ es el punto de partida para la reorganización en la oclusión aunque su definición haya estado sujeta a cambios (Keshvad y Winstanle, 2001) (30).

\section{CONCLUSIÓN}

El glosario de términos prostodónticos debería ser considerado como referencia estándar para evitar confusión y diversidad en la RC.

\section{BIBLIOGRAFÍA}

1. Jasinevicius TM y cols. Centric Relation Definitions Taught in 7 Dental Schools: Results of faculty and Student Surveys. J Prosthodontics 2000;9(2):87-94.

2. McCollum BB. The mandibular Hinge Axis and a method of locating it. J Prosthet Dent 1960;10(3): 428-35.

3. Lucia VO.Centric Relation: theory and practice. J Prosthet Dent 1960;10(5):849-56.

4. Academy of Prosthodontics. Glossary of Prosthodontics Terms, ed 1. J Prosthet Dent 1956;6(1):534.

5. Academy of Prosthodontics. Glossary of Prosthodontics Terms, ed 5. J Prosthet Dent 1987;58 (1):713-62.

6. Academy of Prosthodontics. Glossary of Prosthodontics Terms, ed 6. J Prosthet Dent 1994; 71(1): 41-112.

7. Academy of Prosthodontics. Glossary of Prosthodontics Terms, ed 7. J Prosthet Dent 1999;81(1): 39-110. 
8. Academy of Prosthodontics. Glossary of Prosthodontics Terms, ed 8. J Prosthet Dent 2005;94(1): 10-92.

9. Dawson P. Optimun TMJ condyle position in clinical practice. Int J. Periodontics and Restorative Dent 1985;5(3):11-32.

10. Ash MM, Ramfjord S. Oclusión. $4^{\mathrm{a}}$ Ed México: McGraw-Hill Interamericana 1996, p 70-77.

11. Christensen GJ. Is occlusion becoming more confunsing? J Am Dent Assoc 2004;135(6):76770.

12. Davies S, Gray RMJ. What is occlusion? British Dental Journal 2001;191(5):235-8.

13. Lucia VO. The Fundamentals of oral physiology and their practical application in the securing and reproducing of records to be used in restorative dentistry. J Prosthet Dent 1953;3(2): 213-31.

14. Calagna BA y cols. Influence of neuromuscular conditioning on centric relation registrations. J Prosthet Dent 1973;30(4): 598-604.

15. Posselt ULF. Fisiología de la oclusión y rehabilitación. $2^{a}$ edición España: Ed Jims, 1973.

16. Kantor ME y cols. Centric relation recording techniques: a comparative investigation. J Prosthet Dent 1972;28(6) 593-600.

17. Long $\mathrm{JH}$. Location of the terminal hinge axis by intraoral means. J. Prosthet Dent 1970;23(1): $11-24$.

18. Ash MM, Ramfjord SP. Oclusión funcional. $1^{\mathrm{a}}$ edición México: Nueva Ed Interamericana S.A. 1984.

19. Woelfel JB. New device for accurately recording centric relation. J Prosthet Dent 1986;56(6): 716-27.
20. Senchermen G, Echeverri E. Neurofisiología de la oclusión. $2^{a}$ edición aumentada Colombia: Ed Monserrate 1995.

21. Jankelson RR. Neuromuscular dental diagnosis and treatment. Ishiyaku Euroamericana. Inc. 1990.

22. Jankelson B. The Myo-monitor: its use and abuse (I). Quintessence Int 1978; 9(2): 47-52.

23. Long JH. Locating centric relation with a leaf gauge. J Prosthet Dent 1973;29(6):608-10.

24. Lucia VO. A technique for recording centric relation. J Prosthet Dent 1964;14(3):492-505.

25. Celenza FV. The centric position: replacement and character. J Prosthet Dent 1973;30(4):591-8.

26. Glickman I y cols. Telemetric comparison of centric relation and centric occlusion reconstructions. J Prosthet Dent 1974;31(5):527-36.

27. Rieder CE. The prevalence and magnitude of mandibular displacement in a survey population. J Prosthet Dent 1978;39(3):324-9.

28. Schuyler $\mathrm{CH}$. Fundamental principles in the correction of occlusal disharmony, natural and artificial. J Am Dent Assoc 1935;22:1193-202.

29. Mann AW, Pankey LD. Concepts of Occlusion. Dent Clin North Am 1963;7(3):621-36.

30. Keshvad A, Winstanley RB. An appraisal of literatura on centric relation. Part III. J Oral Rehabilitation 2001;28(1):55-63.

\section{CORRESPONDENCIA}

Ana Orozco Varo

Urb. Macarena tres huertas Bq. 1, $6^{\circ} \mathrm{B}$

41009 Sevilla. España

E-mail:anaorozcovaro@gmail.com 